\title{
Resgatando anonimatos
}

\author{
Harley E. A. Bicas, Cristina Muccioli, Mauro Goldchmit, \\ Mauro S. Q. Campos, Samir Jacob Bechara, Vital Paulino Costa
}

Além do quadro normal de seus colaboradores editoriais, analistas da conveniência de publicação dos artigos que lhes são encaminhados, os Arquivos Brasileiros de Oftalmologia freqüentemente se vêem obrigados a requisitar tais serviços a outras pessoas. De fato, rotineiramente distribuindo cada trabalho recebido ao exame de pelo menos dois assessores (e às vezes mais, na dependência do que seja por eles respondido), nosso sistema de revisão poderia sobrecarregá-los, se fossem reiteradamente acionados sempre os mesmos nomes: apesar de numerosos, são também muitos os artigos que nos chegam, todos examinados no conteúdo científico e na forma de apresentação. Além disso, tais assessores são ainda, costumeiramente, chamados a outras atividades do C.B.O. A necessidade de suplementação dessa lista é, portanto, ineludível.

O trabalho de revisão, finalmente, parece estar sendo compreendido entre nós. Principalmente pelos autores, que agora já tomam as freqüentes críticas e reparos como uma condição com que podem melhorar seus trabalhos. Afinal, ninguém é tão perfeito que não possa ser ajudado ou contestado, nem um trabalho tão irrepreensível ao qual não caiba qualquer sugestão de aperfeiçoamento. Felizmente já vai longe a atitude imatura e imprudente dos que tomavam todo questionamento aos seus escritos (cujas autorias, tanto de uns quanto de outros, são conservadas em sigilo) como ofensa pessoal e arrogância do examinador. Claro que este também, humano, pode errar nos conceitos emitidos ou se exceder em expressões impróprias; mas é por isso que se busca a pluralidade de opiniões e o julgamento (também falível) dos editores. De qualquer modo têm imperado, como regra, a elegância e a paciência de nossos autores.

Mas, também, o trabalho de revisão vem sendo bem desempenhado pela maioria de nossos colaboradores editoriais. Oferecem análises, por vezes tão extensas quanto os próprios artigos examinados e contribuem, genuinamente, para que eles possam ser corrigidos e melhorados; ou recusados (para o bem de seus autores, evitando-lhes o constrangimento da divulgação pública e o da perpetuação impressa de seus erros). Já são também raros os que, ainda, enganam-se em sua função ao emitir uma sentença sumária, ainda que favorável: "Artigo bom, publique-se". O revisor não é um juiz, nem sua pretensa benevolência ajuda ao autor. Também são raros os que não respeitam o prazo de devolução das análises pedidas, não se dando conta que este atraso resulta numa injusta demora para a eventual publicação dos artigos. O trabalho de análise editorial de um artigo é tão importante que os A.B.O. propõem desde há já algum tempo - adiantando-se a uma tendência que começa a se difundir internacionalmente entre editores - a oportunidade de ser ele transparentemente indicado. Claro que tal menção fica inteiramente subordinada ao arbítrio do próprio revisor. Mas é preciso reconhecer que a maioria ainda se sente mais confortável em permanecer no anonimato. Quem sabe? talvez, num futuro ainda remoto, com uma comunidade científica madura e responsável, essa contribuição editorial possa vir a ser, ideal e publicamente, reconhecida; como se o revisor fosse não apenas um analista mas, sobretudo, um avalista do artigo.

É a tais pessoas, tão operosas quanto desinteressadas do recebimento de favores e sabidamente conhecedoras dos assuntos sobre os quais são convidadas a opinar, que queremos, novamente, homenagear. A grandeza de nossa revista e o respeito de que ela goza em nossa comunidade oftalmológica, assim como no âmbito da ciência nacional e internacional são, também, a elas devidos. Tirando-as do anonimato com que cooperaram com os A.B.O., nossos agradecimentos sinceros e os aplausos - aos analistas de trabalhos no ano de 2001 que não estiveram em nossa lista de Conselheiros Editoriais:

\section{A. Duarte}

Abelardo de Souza Couto Jr.

Acácio Alves Souza Lima Filho

Acácio Muralha Neto

Adamo Lui Neto

Adriana Berezowsky

Adriana dos Santos Forsseto

Akyoshi Oshima

Alexandre Chater Taleb

Amélia Kamegasawa

Ana Estela B. P. Ponce Sant'anna

Ana Maria Marcondes

Ana Maria Noriega Petrilli

Ana Teresa Ramos Moreira

André Correa Maia de Carvalho

André Marcelo Vieira Gomes

Arnaldo Furman Bordon

Augusto Paranhos Junior

Breno Barth

Carlos Akira Omi

Carlos Eduardo Leite Arieta

Carlos Eduardo N. Pavesio

Carlos Filipe C.C.Chicani

Carlos Rubens Lucas de Figueiredo

Carmo Mandia Jr. 
Célia Regina Nakanami

César Lipener

Christiane Rolim de Moura

Cinthia Oyama

Cláudia Maria Francesconi

Clélia Maria Erwenne

Cleusa Coral Ghanem

Cristina Maria Bittencourt Garrido

Daniel Madeira

Danilo Sone Soriano

Denise Fornazari de Oliveira

Edmea Rita Temporini

Edmundo Frota de Almeida Sobrinho

Edson Shizuo Mori

Eduardo Marcelo Moron de Andrade

Eduardo Melani Rocha

Élcio Hideo Sato

Eliana Aparecida Forno Velasco

Emerson Francisco Pereira das Neves

Fábio João Zamboni

Fausto Uno

Flávio Augusto Paranhos

Flávio Jaime da Rocha

Francisco de Assis Cordeiro Barbosa

Hélia Soares Angotti

Ítalo Mundialino Marcon

Javier Enrique Yugart Larrea

João Alberto Holanda de Freitas

João Amaro Ferrari Silva

João Antonio Prata Jr.

João Carlos de M. Gonçalves

Joaquim Marinho Queiroz Junior

John Helal

José Álvaro Pereira Gomes

José Antonio de Almeida Milani

José Augusto Cardillo

José Carlos Eudes Carani

José Guilherme de C. Pecego

José Luis Teixeira Ferreira Pires

José Paulo Cabral Vasconcelos

Jose Ricardo de Abreu Reggi

José Wilson Cursino

Joyce Hisae Yamamoto

Juliana Maria Ferraz Sallum

Keila Miriam M. de Carvalho

Laurentino Biccas Neto

Leiria de Andrade Neto

Leonardo Akaishi

Liane Maria de O. Ventura

Lúcia Miriam Dumont Lucci

Luciana Lucci

Luciana Peixoto dos Santos

Luciene Barbosa de Sousa

Luís Paves

Luiz Antonio Vieira

Magno Antonio Ferreira

Márcia Beatriz Tartarella

Márcia Regina Issa Salomão Libanio

Márcia Rowen

Marco Aurélio Lana Peixoto
Marco César Helena

Marcos Wilson Sampaio

Maria Carmen Menezes Santos Torres

Maria Cristina Barbosa de Souza

Maria Cristina Martins

Maria Cristina Nishiaki Dantas

Maria Rosa Bet de Moraes e Silva

Maria Tereza B. C. Bonanomi

Marivaldo Castro de Oliveira

Marta Beatriz C. F. Sartori

Marta Maria Motomo Chojniak

Maurício Bastos Pereira

Maurício Della Paolera

Nelson Alexandre Sabrosa

Newton Kara José Jr.

Nilo Holzchuh

Nilson de Mello e Oliveira

Nilva Simeren Bueno Moraes

Niro Kasahara

Norma Allemann

Norma Helen Medina

Nubia Cristina de Freitas Maia

Osvaldo Gianotti Filho

Oswaldo Tella Jr.

Paiva Gonçalves Neto

Paola Zucchi

Patrícia Ioschpe Gus

Paula Boturão de Almeida

Paulo Góis Manso

Paulo Henrique de A. Morales

Paulo Sérgio Moraes Barros

Regina Halfeld Furtado de Mendonça

Renato Ambrósio Junior

Ricardo Belfort

Ricardo Lewinsky

Ricardo Uras

Roberto Freda

Roberto Pedrosa Galvão Filho

Rosa Maria Graziano

Rosana Nogeira Pires da Cunha

Rosane da Cruz Ferreira

Ruth Alice R. Schor

Ruth Miyuki Santo

Saly Moreira

Samuel Rymer

Sandra Maria C. Beer

Seiji Hayashi

Sérgio Luiz G. Pimentel

Sérgio Vanetti Burnier

Sidney Júlio de Faria e Sousa

Solange Rios Salomão

Sung Bok Cha

Teruo Aihara

Tomás F. Scalamandré Mendonça

Vera Lúcia D. Monte Mascaro

Vera Regina C. Castanheira

Walter Yukihiko Takahashi

Walton Nosé

Wesley Ribeiro Campos

Zélia Maria da Silva Correa 